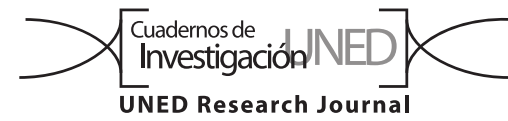

\title{
Producción de pepinillo (Cucumis sativus) en un ambiente protegido: evaluación de dos genotipos
}

\author{
José Aníbal Cruz-Coronado' $\&$ José Eladio Monge-Pérez² \\ 1. Universidad de Costa Rica, San José, Costa Rica; cruzcsanibal@gmail.com; (iD https://orcid.org/0000-0002-8290-3443 \\ 2. Universidad de Costa Rica, Estación Experimental Agrícola Fabio Baudrit Moreno, Alajuela, Costa Rica; melonescr@yahoo.com.mx; \\ (D) https://orcid.org/0000-0002-5384-507X
}

Recibido 20-VI-2019 • Corregido 10-IX-2019 • Aceptado 30-IX-2019

DOI: https://doi.org/10.22458/urj.v11i3.2720

\begin{abstract}
Production of mini cucumber (Cucumis sativus) grown under greenhouse conditions: evaluation of two genotypes". Introduction: Mini cucumber is an interesting new crop in Costa Rica. Objective: to evaluate yield and quality of two mini parthenocarpic cucumber genotypes (Larino and 22-20-781) grown under greenhouse conditions at the Agricultural Experimental Station Fabio Baudrit Moreno located in Alajuela, Costa Rica. Methods: The crop was planted on coconut fiber as substrate, pruned to one stem per plant, and managed with fertigation. Results: Flowering started 16 days after transplant (dat) and harvest started 26 dat, for both genotypes. Larino yielded fruits with higher length $(12,19 \mathrm{~cm})$, but lower diameter $(41,44 \mathrm{~mm})$ and lower percentage of total soluble solids $\left(3,00{ }^{\circ} \mathrm{Brix}\right)$, compared to 22-20-781 genotype. There were no significant differences in fruit weight among genotypes (137,37 to $140,38 \mathrm{~g})$. First quality fruits showed higher length and diameter, as well as a higher percentage of total soluble solids, than second quality fruits. Conclusions: Larino genotype obtained the highest total number of fruits per plant $(98,63)$, and the highest commercial $\left(29,55 \mathrm{~kg} / \mathrm{m}^{2}\right)$ and first quality yield $\left(18,60 \mathrm{~kg} / \mathrm{m}^{2}\right)$, so it is considered the best adapted to the conditions of the trial.
\end{abstract}

Key words: Cucumis sativus, genotypes, mini cucumber, greenhouse, yield, quality.
RESUMEN: Introducción: El pepinillo es un cultivo nuevo e interesante en Costa Rica. Objetivo: evaluar el rendimiento y la calidad de dos genotipos de pepinillo partenocárpico (Larino y 22-20-781), cultivados bajo condiciones de invernadero en la Estación Experimental Agrícola Fabio Baudrit Moreno, en Alajuela, Costa Rica. Métodos: El cultivo se manejó con fertirrigación, en sustrato de fibra de coco, y con poda a un tallo por planta. Resultados: La floración inició a los 16 días después del trasplante (ddt), y la cosecha inició a los $26 \mathrm{ddt}$, para ambos genotipos. Larino presentó frutos con mayor longitud $(12,19 \mathrm{~cm})$, pero menor diámetro $(41,44 \mathrm{~mm})$ y menor porcentaje de sólidos solubles totales (3,00 ${ }^{\circ}$ Brix), en comparación con el genotipo 22-20-781. No se presentaron diferencias significativas en el peso del fruto entre ambos genotipos (entre 137,37 y 140,38g). Los frutos de primera calidad mostraron mayor longitud y diámetro que los de segunda calidad y de rechazo. Los frutos de primera calidad obtuvieron un mayor porcentaje de sólidos solubles totales que los de segunda calidad. Conclusiones: El genotipo Larino obtuvo el mayor número total de frutos por planta $(98,63)$, y el mayor rendimiento comercial $\left(29,55 \mathrm{~kg} / \mathrm{m}^{2}\right)$ y de primera calidad $\left(18,60 \mathrm{~kg} / \mathrm{m}^{2}\right)$, por lo que se considera el mejor adaptado a las condiciones en que se desarrolló la investigación.

Palabras clave: Cucumis sativus, genotipos, pepinillo, invernadero, rendimiento, calidad.
El pepino, Cucumis sativus L., pertenece a la familia de plantas cucurbitáceas, y es una hortaliza que se cultiva en condiciones tropicales y subtropicales alrededor del mundo; es una especie nativa del norte de India (Kapuriya, y otros, 2017); la planta es herbácea, anual, de hábito rastrero o trepador cuando se le proporciona un tutorado, y de crecimiento indeterminado (Reche, 2011).

Las condiciones óptimas de temperatura para el desarrollo del pepino se encuentran en un ámbito de 18 a 28 ${ }^{\circ} \mathrm{C}$ (Bojacá \& Monsalve, 2012); por otra parte, la FAO indica que el pepino se desarrolla de manera óptima entre 18 a $25^{\circ} \mathrm{C}$, y una humedad relativa entre 70 y $90 \%$ (FAO, 2002).

El pepino se adapta a climas cálidos y templados, y se cultiva desde las zonas costeras hasta los $1200 \mathrm{~m}$ sobre el nivel del mar. Por encima de $40^{\circ} \mathrm{C}$ el crecimiento se detiene, y lo mismo sucede con temperaturas inferiores a $14^{\circ} \mathrm{C}$. Es una planta con elevados requerimientos de humedad debido a su gran superficie foliar, siendo la humedad relativa óptima durante el día de 60-70\%, y durante la noche de $70-90 \%$ (Casaca, 2005). 
Las temperaturas muy altas durante el periodo de floración disminuyen la viabilidad del polen (Haifa, 2011), por lo que la producción se ve afectada. Asimismo, se considera que las temperaturas de 20 a $30^{\circ} \mathrm{C}$ durante el día apenas tienen incidencia sobre la producción; a mayor temperatura, hasta $25{ }^{\circ} \mathrm{C}$, se alcanza la máxima producción precoz, pero por encima de $30^{\circ} \mathrm{C}$ se pueden observar desequilibrios en las plantas (Vasco, 2003).

Según su uso, los pepinos se pueden clasificar en: pepino para encurtido (procesamiento); pepino de mesa o para ensalada (consumo fresco); cultivares de invernadero; armenio; oriental (japonés o asiático); Beit Alfa (persa); y europeo (Haifa, 2011). Otra de las clasificaciones utiliza como criterio el origen, como es el caso de los tipos holandés y francés (también llamados europeos), y el tipo asiático. Un criterio adicional es el tamaño del fruto: largo (tipo holandés), mediano (tipo americano o "slicer", y francés), y pequeño (tipo Beit Alpha, mini, o pepinillo).

Los pepinos híbridos tipo Beit-Alfa (pepinillo) son originarios de Israel, y están distribuidos a nivel mundial (Shaw, Cantliffe, Rodríguez, Taylor, \& Spencer, 2000); producen varios frutos por nudo, lo que aumenta el rendimiento de frutos, al compararlo con los tipo holandés que solo producen un fruto por nudo (Crosby, 2008); se utilizan para invernadero, y al igual que el tipo holandés, tienen la cáscara delgada, y se deben proteger de los insectos y la deshidratación; los frutos son relativamente delgados, de cáscara lisa y sin espinas, de color verde claro uniforme, y se cosechan entre $8-13 \mathrm{~cm}$ de longitud (Johnny's Selected Seeds, 2014).

El pepino es una hortaliza de alta demanda en países desarrollados, principalmente para la industria de vegetales procesados o para encurtido, aunque también para consumo fresco (Musmade \& Desai, 2003). De ahí la importancia de evaluar nuevos materiales de pepino que les sirvan a los productores para mantenerse en el mercado nacional e incluso incursionar en la actividad de exportación, con variedades que sean vigorosas, precoces, de altos rendimientos y de buena calidad.

El objetivo de esta investigación fue evaluar el rendimiento y la calidad de dos genotipos de pepinillo partenocárpico, cultivados bajo ambiente protegido en condiciones hidropónicas, en Alajuela, Costa Rica.

\section{MATERIALES Y MÉTODOS}

Se sembraron dos genotipos híbridos de pepinillo (Cucumis sativus L.) partenocárpico: Larino, de la casa comercial Rijk Zwaan; y 22-20-781, de la casa comercial Pandia Seeds. El cultivo se realizó en condiciones hidropónicas, en el invernadero de Hortalizas de la Estación Experimental Agrícola Fabio Baudrit Moreno (EEAFBM), ubicada en Barrio San José de Alajuela, Costa Rica, a una altitud de $883 \mathrm{msnm}$.

El cultivo se realizó en sacos plásticos rellenos con fibra de coco, de $1 \mathrm{~m}$ de largo, $20 \mathrm{~cm}$ de ancho y $15 \mathrm{~cm}$ de altura. La distancia de siembra fue de $25 \mathrm{~cm}$ entre plantas, y de $1,54 \mathrm{~m}$ entre hileras, para una densidad de 2,60 plantas $/ \mathrm{m}^{2}$. Las plantas se manejaron a un solo tallo, eliminando todos los tallos secundarios. Las labores de amarre de la planta, deshijas y deshojas se realizaron en forma periódica. Se eliminaron los primeros cuatro frutos de cada planta, para lograr una cosecha más uniforme.

Durante este ensayo, las condiciones ambientales dentro del invernadero fueron las siguientes: la temperatura diurna promedio fue de $27,1^{\circ} \mathrm{C}$, con un valor mínimo promedio de $20,7^{\circ} \mathrm{C}$, y un máximo promedio de $33,4^{\circ} \mathrm{C}$; mientras que la temperatura nocturna promedio fue de $21,4^{\circ} \mathrm{C}$, con un valor mínimo promedio de $19,8^{\circ} \mathrm{C}$ y un máximo promedio de $23,9^{\circ} \mathrm{C}$. La humedad relativa promedio durante el día fue de $72,3 \%$, y durante la noche fue de $89,2 \%$. La radiación fotosintéticamente activa diurna promedio fue de $494,0 \mathrm{~W} / \mathrm{m}^{2}$.

El almácigo se sembró el 3 de julio de 2015; se usaron bandejas de 98 celdas, y se utilizó turba ("peat moss") como sustrato. El trasplante se realizó 12 días después de la siembra, cuando las plántulas tenían una hoja verdadera. El período de cultivo abarcó desde el 15 de julio hasta el 20 de octubre de 2015, es decir, hasta los 97 días después del trasplante (ddt).

El suministro hídrico y la fertilización se hicieron mediante un sistema de fertirriego automático controlado por un dispositivo marca iGrow 1400. El plan de fertirriego se presenta en el Cuadro 1.

Se clasificó la cosecha según tres categorías de calidad (Cuadro 2).

CUADRO 1

Plan de fertirriego utilizado para la producción de genotipos de pepinillo

\begin{tabular}{lcccccccccccc}
\multicolumn{11}{c}{ Etapa del cultivo } & \multicolumn{11}{c}{ Concentración del nutriente $(\mathrm{mg} / \mathrm{l})$} \\
& $\mathrm{N}$ & $\mathrm{P}$ & $\mathrm{K}$ & $\mathrm{Ca}$ & $\mathrm{Mg}$ & $\mathrm{S}$ & $\mathrm{Cu}$ & $\mathrm{Fe}$ & $\mathrm{Zn}$ & $\mathrm{Mn}$ & Mo & $\mathrm{B}$ \\
0-15 ddt & 150 & 53 & 240 & 165 & 40 & 50 & 0,16 & 2,9 & 0,3 & 0,6 & 0,9 & 0,8 \\
15-30 ddt & 161 & 53 & 265 & 175 & 50 & 50 & 0,16 & 2,9 & 0,3 & 0,6 & 0,9 & 0,8 \\
30 ddt en adelante & 172,5 & 53 & 290 & 175 & 55 & 50 & 0,16 & 2,9 & 0,3 & 0,6 & 0,9 & 0,8 \\
\hline
\end{tabular}


CUADRO 2

Parámetros de calidad de pepino utilizados en el ensayo

\begin{tabular}{llcc}
\multicolumn{1}{c}{ Parámetro } & Categoría de calidad & Rechazo \\
& Primera & Segunda & Severa \\
Deformación de frutos & Ausente & Leve & Mayor a $1 \mathrm{~cm}^{2}$ \\
Daños en la cáscara del fruto & Ausente & Menor o igual a $1 \mathrm{~cm}^{2}$ & Mayola \\
\hline
\end{tabular}

Se evaluaron las siguientes variables:

a. Edad al inicio de floración (ddt): Esta variable se midió en días después del trasplante, cuando el $50 \%$ de las plantas mostraban al menos una flor completamente abierta.

b. Edad al inicio de cosecha (ddt): Esta variable se registró en días después del trasplante al realizar la primera cosecha, cuando los frutos habían alcanzado un estado de desarrollo óptimo para la comercialización.

c. Número de frutos por planta: Se contabilizó el número total de frutos producidos por parcela, y se dividió entre el número de plantas de la parcela.

d. Longitud del fruto $(\mathrm{cm})$ : Se evaluó esta característica en 20 frutos de cada categoría de calidad en cada repetición, y se obtuvo el promedio; se determinó con una cinta métrica marca Assist 32G-8025, con una capacidad de $800,0 \pm 0,1 \mathrm{~cm}$.

e. Diámetro del fruto $(\mathrm{mm})$ : Se evaluó esta característica en la parte media de 20 frutos de cada categoría de calidad por repetición, y se obtuvo el promedio; se midió con un calibrador digital marca Mitutoyo modelo CD-6"CS, con una capacidad de 15,00 $\pm 0,01 \mathrm{~cm}$.

f. Porcentaje de sólidos solubles totales ('Brix): Se evaluó esta característica a partir de un macerado de la pulpa y la placenta del fruto, en 20 frutos por cada repetición y categoría de calidad, y se obtuvo el promedio; se midió con un refractómetro manual marca BOECO, con una capacidad de $0-32,0 \pm 0,2^{\circ}$ Brix.

g. Peso del fruto (g): Se midió el peso total de los frutos producidos por repetición y categoría de calidad, y se dividió entre el número total de frutos; se determinó por medio de una balanza electrónica marca OCONY UWE HGM-20, con una capacidad de $20000 \pm 1 \mathrm{~g}$.

h. Rendimiento $\left(\mathrm{kg} / \mathrm{m}^{2}\right)$ : Se estimó a partir del peso de la producción obtenida por parcela y de la densidad de siembra, para cada categoría de calidad. Se consideró como rendimiento comercial la suma de las categorías de primera y segunda calidad, y como rendimiento total la suma de las tres categorías de calidad.

Se utilizó un diseño experimental irrestricto al azar, con 2 tratamientos (genotipos), y cuatro repeticiones.
Cada parcela contó con 8 plantas (2 sacos), y todos los datos se obtuvieron a partir de los frutos totales producidos en dicha parcela. Para las variables cuantitativas se realizó un análisis estadístico de variancia, y se utilizó la prueba de LSD Fisher con una significancia de 5\% para determinar diferencias entre los tratamientos.

Ética, conflicto de intereses y declaración de financiamiento: Los autores declaran haber cumplido con todos los requisitos éticos y legales pertinentes, tanto durante el estudio como en el manuscrito; que no hay conflictos de interés de ningún tipo, y que todas las fuentes financieras se detallan plena y claramente en la sección de agradecimientos. Asimismo, están de acuerdo con la versión editada final del documento. El respectivo documento legal firmado se encuentra en los archivos de la revista.

\section{RESULTADOS}

Con relación a la fenología del cultivo, en el caso de ambos genotipos el inicio de la floración (antesis) se produjo a los $16 \mathrm{ddt}$, y el inicio de la cosecha sucedió a los 26ddt.

El genotipo 22-20-781 presentó una longitud del fruto significativamente menor $(11,54 \mathrm{~cm})$, en comparación al Larino $(12,19 \mathrm{~cm})$ (Cuadro 3); esta diferencia también fue significativa para cada categoría de calidad. Además, en términos generales, los frutos de primera calidad mostraron una mayor longitud que los de segunda calidad y de rechazo; y los de segunda calidad obtuvieron también una mayor longitud que los de rechazo.

Larino obtuvo un diámetro del fruto significativamente menor $(41,44 \mathrm{~mm})$, en contraste con el genotipo 22 20-781 (44,87mm) (Cuadro 4); esta diferencia también fue significativa para cada categoría de calidad. Por otra parte, a nivel general, los frutos de primera calidad presentaron un mayor diámetro que los de segunda calidad y de rechazo; $y$ asimismo los de segunda calidad mostraron un mayor diámetro que los de rechazo. 
CUADRO 3

Longitud del fruto $(\mathrm{cm})$ de los genotipos de pepinillo evaluados

\begin{tabular}{|c|c|c|c|c|}
\hline \multirow{2}{*}{ Genotipo } & \multicolumn{4}{|c|}{ Longitud del fruto $(\mathrm{cm})$, según categoría de calidad } \\
\hline & Total & Primera & Segunda & Rechazo \\
\hline $22-20-781$ & $11,54 \mathrm{a}$ & $12,20 \mathrm{a}$ & $11,78 \mathrm{a}$ & 10,64 a \\
\hline Larino & $12,19 b$ & $12,96 \mathrm{~b}$ & $12,22 b$ & $11,40 \mathrm{~b}$ \\
\hline \multicolumn{5}{|l|}{ Categoría de calidad } \\
\hline Primera & $12,58 \mathrm{c}$ & & & \\
\hline Segunda & $12,00 \mathrm{~b}$ & & & \\
\hline Rechazo & $11,02 \mathrm{a}$ & & & \\
\hline
\end{tabular}

*Valores con una letra en común no son significativamente diferentes, según prueba LSD Fisher $(p \leq 0,05)$.

CUADRO 4

Diámetro del fruto $(\mathrm{mm})$ de los genotipos de pepinillo evaluados

\begin{tabular}{lcccc}
\multicolumn{1}{c}{ Genotipo } & \multicolumn{4}{c}{ Diámetro del fruto $(\mathrm{mm})$, según categoría de calidad } \\
Larino & Total & Primera & Segunda & Rechazo \\
$22-20-781$ & $41,44 \mathrm{a}$ & $45,59 \mathrm{a}$ & $41,17 \mathrm{a}$ & $37,57 \mathrm{a}$ \\
Categoría de calidad & $44,87 \mathrm{~b}$ & $49,01 \mathrm{~b}$ & $45,47 \mathrm{~b}$ & $40,12 \mathrm{~b}$ \\
Primera & & & & \\
Segunda & $47,30 \mathrm{c}$ & & \\
Rechazo & $43,32 \mathrm{~b}$ & & & \\
\hline
\end{tabular}

* Valores con una letra en común no son significativamente diferentes, según prueba LSD Fisher $(p \leq 0,05)$.

El genotipo Larino presentó la mayor producción de frutos, tanto para la cantidad total $(98,63$ frutos por planta), como para la categoría de primera calidad $(44,16$ frutos por planta) (Cuadro 5). En las categorías de segunda calidad y de rechazo no se presentaron diferencias significativas entre genotipos.

Larino obtuvo el mayor valor en el peso del fruto de primera calidad $(168,12 \mathrm{~g})$, en comparación con el genotipo 22-20-781 (162,23g) (Cuadro 6); en cuanto al peso total del fruto, no se presentaron diferencias significativas entre genotipos, ni tampoco en los frutos de segunda calidad ni en los de rechazo.
En cuanto al rendimiento de primera calidad, se presentaron diferencias significativas entre genotipos, y Larino obtuvo una mayor producción $\left(18,60 \mathrm{~kg} / \mathrm{m}^{2}\right)$ (Cuadro 7). Por otra parte, a pesar de que en el rendimiento de segunda calidad y rechazo no se hallaron diferencias significativas, en el rendimiento comercial y el total sí se presentaron diferencias, y el genotipo Larino fue el más productivo con $29,55 \mathrm{~kg} / \mathrm{m}^{2}$ en rendimiento comercial y $36,58 \mathrm{~kg} / \mathrm{m}^{2}$ en rendimiento total, en comparación con $24,66 \mathrm{~kg} / \mathrm{m}^{2}$ y $30,50 \mathrm{~kg} / \mathrm{m}^{2}$ producidos por el genotipo 22-20-781, en rendimiento comercial y total, respectivamente.

CUADRO 5

Número de frutos por planta de los genotipos de pepinillo evaluados

\begin{tabular}{lcccc}
\multicolumn{1}{r}{ Genotipo } & \multicolumn{4}{c}{ Número de frutos por planta, según categoría de calidad } \\
& Total & Primera & Segunda & Rechazo \\
$22-20-781$ & $83,07 \mathrm{a}$ & $33,88 \mathrm{a}$ & $28,66 \mathrm{a}$ & $20,53 \mathrm{a}$ \\
Larino & $98,63 \mathrm{~b}$ & $44,16 \mathrm{~b}$ & $29,72 \mathrm{a}$ & $24,75 \mathrm{a}$ \\
\hline
\end{tabular}

* Valores con una letra en común no son significativamente diferentes, según prueba LSD Fisher $(p \leq 0,05)$. 
CUADRO 6

Peso del fruto $(\mathrm{g})$ de los genotipos de pepinillo evaluados

\begin{tabular}{lcccc} 
& \multicolumn{4}{c}{ Peso del fruto $(\mathrm{g})$, según categoría de calidad } \\
Genotipo & Total & Primera & Segunda & Rechazo \\
$22-20-781$ & $137,37 \mathrm{a}$ & $162,23 \mathrm{a}$ & $140,20 \mathrm{a}$ & $109,68 \mathrm{a}$ \\
Larino & $140,38 \mathrm{a}$ & $168,12 \mathrm{~b}$ & $142,71 \mathrm{a}$ & $110,32 \mathrm{a}$ \\
\hline
\end{tabular}

*Valores con una letra en común no son significativamente diferentes, según prueba LSD Fisher $(p \leq 0,05)$.

CUADRO 7

Rendimiento $\left(\mathrm{kg} / \mathrm{m}^{2}\right)$ de los genotipos de pepinillo evaluados

\begin{tabular}{lccccc}
\multicolumn{5}{r}{ Genotipo } & \multicolumn{5}{c}{ Rendimiento $\left(\mathrm{kg} / \mathrm{m}^{2}\right)$, según categoría de calidad } \\
& Total & Comercial & Primera & Segunda & Rechazo \\
$22-20-781$ & $30,50 \mathrm{a}$ & $24,66 \mathrm{a}$ & $14,23 \mathrm{a}$ & $10,43 \mathrm{a}$ & $5,84 \mathrm{a}$ \\
Larino & $36,58 \mathrm{~b}$ & $29,55 \mathrm{~b}$ & $18,60 \mathrm{~b}$ & $10,95 \mathrm{a}$ & $7,03 \mathrm{a}$ \\
\hline
\end{tabular}

*Valores con una letra en común no son significativamente diferentes, según prueba LSD Fisher $(p \leq 0,05)$.

CUADRO 8

Porcentaje de sólidos solubles totales ( $\left.{ }^{\circ} \mathrm{Brix}\right)$ del fruto de los genotipos de pepinillo evaluados

\begin{tabular}{|c|c|c|c|c|}
\hline \multirow{2}{*}{ Genotipo } & \multicolumn{4}{|c|}{ Porcentaje de sólidos solubles totales ('Brix), según categoría de calidad } \\
\hline & Total & Primera & Segunda & Rechazo \\
\hline Larino & $3,00 \mathrm{a}$ & $3,02 \mathrm{a}$ & $2,99 \mathrm{a}$ & $3,00 \mathrm{a}$ \\
\hline 22-20-781 & $3,09 \mathrm{~b}$ & $3,15 \mathrm{~b}$ & $3,03 \mathrm{a}$ & $3,10 \mathrm{~b}$ \\
\hline \multicolumn{5}{|l|}{ Categoría de calidad } \\
\hline Primera & $3,08 \mathrm{~b}$ & & & \\
\hline Segunda & $3,01 \mathrm{a}$ & & & \\
\hline Rechazo & $3,05 \mathrm{ab}$ & & & \\
\hline
\end{tabular}

*Valores con una letra en común no son significativamente diferentes, según prueba LSD Fisher $(p \leq 0,05)$.

El genotipo 22-20-781 obtuvo un mayor porcentaje de sólidos solubles totales $\left(3,09^{\circ}\right.$ Brix), en comparación al Larino (3,00 ${ }^{\circ}$ Brix) (Cuadro 8); esta diferencia también fue significativa para las categorías de primera calidad y rechazo, pero para la categoría de segunda calidad no se presentaron diferencias significativas entre ambos genotipos. Además, en términos generales, los frutos de primera calidad obtuvieron un mayor valor para esta característica que los de segunda calidad.

\section{DISCUSIÓN}

Según diversos investigadores la edad al inicio de cosecha oscila entre 24 y 59ddt para pepinillo (ver cuadro 9 en el Apéndice Digital) (Nomura \& Cardoso, 2000; Cardoso, 2002; Cardoso \& Silva, 2003; Hochmuth et al., 2004; Shaw, Cantliffe, Funes, \& Shine III, 2004; Soleimani,
Ahmadikhah, \& Soleimani, 2009; Arshad, Ali, \& Khan, 2014; Rahil \& Qanadillo, 2015; Arshad, 2017; ChacónPadilla \& Monge-Pérez, 2017). Los resultados hallados en el presente ensayo se encuentran dentro de ese ámbito.

En relación a la longitud del fruto de pepinillo, de acuerdo con varios autores (Shaw, Cantliffe, Rodríguez, Taylor, \& Spencer, 2000; Lamb, Shaw, \& Cantliffe, 2001; Hochmuth et al., 2004; Jasso-Chaverria, Hochmuth, Hochmuth, \& Sargent, 2005; Gómez-López, FernándezTrujillo, \& Baille, 2006; Soleimani, Ahmadikhah, \& Soleimani, 2009; Arshad, Ali, \& Khan, 2014; Galindo et al., 2014; Patil \& Bhagat, 2014; Abu-Zahra \& Ateyyat, 2016; Chacón-Padilla \& Monge-Pérez, 2017; Kapuriya, et al., 2017; Premalatha, Wahundeniya, Weerakkody, \& Wicramathunga, 2006; Arshad, 2017), los valores para esta característica varían entre 12,43 y $21,90 \mathrm{~cm}$ (ver cuadro 10 en el Apéndice Digital); en forma comparativa, los 
resultados obtenidos en la presente investigación se ubicaron por debajo de dicho ámbito, es decir, que los frutos mostraron una menor longitud.

Por otra parte, según otras investigaciones (Shaw, Cantliffe, Rodríguez, Taylor, \& Spencer, 2000; ; Lamb, Shaw, \& Cantliffe, 2001; Hochmuth et al., 2004; JassoChaverria, Hochmuth, Hochmuth, \& Sargent, 2005; Gómez-López, Fernández-Trujillo, \& Baille, 2006; Soleimani, Ahmadikhah, \& Soleimani, 2009; Galindo et al., 2014; Patil \& Bhagat, 2014; Chacón-Padilla \& MongePérez, 2017; Kapuriya et al., 2017), el diámetro del fruto de pepinillo oscila entre 24,40 y 58,00mm (ver cuadro 11 en el Apéndice Digital); los resultados hallados en el presente trabajo se ubicaron dentro de dicho ámbito.

Con respecto a la producción de frutos de pepinillo, de acuerdo con diversos autores (Nomura \& Cardoso, 2000; Shaw, Cantliffe, Rodríguez, Taylor, \& Spencer, 2000; Lamb, Shaw, \& Cantliffe, 2001; Cardoso, 2002; Cardoso \& Silva, 2003; Hochmuthet al., 2004; Shaw, Cantliffe, Funes, \& Shine III, 2004; Premalatha, Wahundeniya, Weerakkody, \& Wicramathunga, 2006; Crosby, 2008; Soleimani, Ahmadikhah, \& Soleimani, 2009; Arshad, Ali, \& Khan, 2014; Patil \& Bhagat, 2014; Sarhan \& Ismael, 2014; Rahil \& Qanadillo, 2015; Abu-Zahra \& Ateyyat, 2016; Arshad, 2017; Chacón-Padilla \& Monge-Pérez, 2017; Kapuriya et al., 2017;), el total producido varía entre 6,00 y 66,80 frutos por planta (ver cuadro 12 en el Apéndice Digital); en comparación con esos datos, los resultados obtenidos en el presente ensayo fueron muy superiores a dicho ámbito, lo que evidencia una mayor prolificidad.

En el caso del pepinillo, al producir frutos de menor tamaño en comparación con los otros tipos de pepino, esto le permite a la planta desarrollar en forma adecuada un mayor número de frutos, a partir de los fotoasimilados producidos. Además, las plantas de Larino y 22-20781 son multifruto, es decir, que producen desde tres hasta cinco frutos por cada nudo, lo que también explica su alta prolificidad.

Se ha informado que la incidencia de alta temperatura (mayor a $30^{\circ} \mathrm{C}$ ) provoca desequilibrios en las plantas de pepino, dando lugar a malformaciones de hojas y frutos defectuosos (Grijalva, Macías, Grijalva, \& Robles, 2011). Este fenómeno probablemente afectó a las plantas en el presente ensayo, ya que las temperaturas máximas dentro del invernadero alcanzaron inclusive $\operatorname{los} 37,4^{\circ} \mathrm{C}$, lo que pudo haber favorecido una alta producción de frutos de calidad de rechazo (entre 20,53 y 24,75 frutos por planta). En este sentido, resultaría interesante estudiar el efecto de la aplicación de la práctica de raleo de frutos (por ejemplo, dejar solamente dos frutos por nudo) en pepinillos multifruto, para determinar si es posible reducir el número de frutos de rechazo por planta, y a la vez aumentar el rendimiento comercial.

De acuerdo con varios investigadores (Nomura \& Cardoso, 2000; Shaw, Cantliffe, Rodríguez, Taylor, \& Spencer, 2000; Lamb, Shaw, \& Cantliffe, 2001; Cardoso, 2002; Cardoso \& Silva, 2003; Hochmuth et al., 2004; Shaw, Cantliffe, Funes, \& Shine III, 2004; GómezLópez, Fernández-Trujillo, \& Baille, 2006; Premalatha, Wahundeniya, Weerakkody, \& Wicramathunga, 2006; Crosby, 2008; Arshad, Ali, \& Khan, 2014; Galindo et al., 2014; Patil \& Bhagat, 2014; Sarhan \& Ismael, 2014; Rahil \& Qanadillo, 2015; Abu-Zahra \& Ateyyat, 2016; Arshad, 2017; Chacón-Padilla \& Monge-Pérez, 2017; Kapuriya et al., 2017;), el peso del fruto de pepinillo oscila entre 44,00 y 330,00 g (ver cuadro 13 en el Apéndice Digital); los resultados hallados en la presente investigación se ubicaron dentro de dicho ámbito.

Según otras investigaciones (Nomura \& Cardoso, 2000; Cardoso, 2002; Cardoso \& Silva, 2003; Hochmuth et al.,2004; Shaw, Cantliffe, Funes, \& Shine III, 2004; GómezLópez, Fernández-Trujillo, \& Baille, 2006; Premalatha, Wahundeniya, Weerakkody, \& Wicramathunga, 2006; Soleimani, Ahmadikhah, \& Soleimani, 2009; Arshad, Ali, \& Khan, 2014; Galindo et al., 2014; Patil \& Bhagat, 2014; Sarhan \& Ismael, 2014; Rahil \& Qanadillo, 2015; Arshad, 2017; Chacón-Padilla \& Monge-Pérez, 2017; Kapuriya et al., 2017), el rendimiento de pepinillo oscila entre 2,38 y $25,25 \mathrm{~kg} / \mathrm{m}^{2}$ (ver cuadro 14 en el Apéndice Digital); en forma comparativa, los resultados obtenidos en el presente trabajo fueron muy superiores a dicho ámbito, lo que demuestra una mayor productividad. Este resultado se explica por la gran prolificidad de los genotipos evaluados.

De acuerdo con otros autores (Gómez-López, Fernández-Trujillo, \& Baille, 2006; Galindo et al., 2014; Chacón-Padilla \& Monge-Pérez, 2017; Kapuriya et al., 2017), el porcentaje de sólidos solubles totales varía entre 2,50 y $5,00^{\circ}$ Brix para pepinillo (ver cuadro 15 en el Apéndice Digital); los resultados hallados en el presente ensayo se ubicaron dentro de dicho ámbito.

Se concluye que el genotipo Larino presentó frutos con mayor longitud, pero menor diámetro y menor porcentaje de sólidos solubles totales, en comparación con el 22-20-781. No se presentaron diferencias significativas en el peso del fruto entre ambos genotipos. Los frutos de pepino de primera calidad mostraron mayor longitud y diámetro que los de segunda calidad y de rechazo. Los frutos de primera calidad obtuvieron un mayor porcentaje de sólidos solubles totales que los de segunda calidad. El genotipo Larino obtuvo el mayor número total de frutos por planta, y el mayor rendimiento comercial y de 
primera calidad, por lo que se considera el mejor adaptado a las condiciones en que se desarrolló la investigación.

\section{AGRADECIMIENTOS}

Los autores agradecen el financiamiento recibido por parte de CONARE, así como de la Universidad de Costa Rica, para la realización de este trabajo. Asimismo, agradecen la colaboración de Mario Monge en la revisión de la traducción del resumen al idioma inglés.

\section{REFERENCIAS}

Abu-Zahra, T. R., \& Ateyyat, M. A. (2016). Effect of various shading methods on cucumber (Cucumis sativus L.) growth and yield production. International Journal of Environment and Sustainability, 5(1), 10-17.

Arshad, I. (2017). Effect of water stress on the growth and yield of greenhouse cucumber (Cucumis sativus L.). PSM Biological Research, 2(2), 63-67.

Arshad, I., Ali, W., \& Khan, Z. A. (2014). Effect of different levels of NPK fertilizers on the growth and yield of greenhouse cucumber (Cucumis sativus) by using drip irrigation technology. International Journal of Research, 1(8), 650-660.

Bojacá, C., \& Monsalve, O. (2012). Manual de producción de pepino bajo invernadero. Bogotá, Colombia: Universidad de Bogotá Jorge Tadeo Lozano.

Cardoso, A. I. (2002). Avaliação de cultivares de pepino tipo caipira sob ambiente protegido em duas épocas de semeadura. Bragantia, 61(1), 43-48.

Cardoso, A. I., \& Silva, N. (2003). Avaliação de híbridos de pepino tipo japonês sob ambiente protegido em duas épocas de cultivo. Horticultura Brasileira, 21(2), 170-175.

Casaca, A. (2005). Guías tecnológicas de frutas y vegetales. El cultivo del pepino. Recuperado de http://www.innovacion. gob.sv/inventa/attachments/article/2286/pepino.pdf

Chacón-Padilla, K., \& Monge-Pérez, J. E. (2017). Evaluación de rendimiento y calidad de tres genotipos de pepino tipo mini (Cucumis sativus L.) cultivados bajo invernadero en Costa Rica, durante la época seca. Tecnología en Marcha, 30(1), 14-26.

Crosby, L. C. (2008). Growth and consumer evaluation of Cucumis sativus L. cultivated in controlled environments (Tesis de maestría).Texas Tech University, Texas, USA.

FAO. (2002). El cultivo protegido en clima mediterráneo: pepino. Recuperado de http://www.fao.org/docrep/005/ s8630s/s8630s08.htm\#bm08..2.4.5

Galindo, F. V., Fortis, M., Preciado, P., Trejo, R., Segura, M. A., \& Orozco, J. A. (2014). Caracterización físico-química de sustratos orgánicos para producción de pepino (Cucumis sativus L.) bajo sistema protegido. Revista Mexicana de Ciencias Agrícolas, 5(7), 1219-1232.

Gómez-López, M. D., Fernández-Trujillo, J. P., \& Baille, A. (2006). Cucumber fruit quality at harvest affected by soilless system, crop age and preharvest climatic conditions during two consecutive seasons. Scientia Horticulturae, 110, 68-78.

Grijalva, R. L., Macías, R., Grijalva, S. A., \& Robles, F. (2011). Evaluación del efecto de la fecha de siembra en la productividad y calidad de híbridos de pepino europeo bajo condiciones de invernadero en el noroeste de Sonora. Biotecnia, 13(1), 29-36.

Haifa. (2011). Nutritional recommendations for cucumber in open fields, tunnels and greenhouse. Recuperado de http:// www.haifa-group.com/files/Guides/Cucumber.pdf

Hochmuth, R. C., Davis, L. L., Laughlin, W. L., Simonne, E. H., Sargent, S. A., \& Berry, A. (2004). Evaluation of twelve greenhouse mini cucumber (Beit Alpha) cultivars and two growing systems during the 2002-2003 winter season in Florida. Research report 2003-2004, University of Florida, IFAS, Florida, EEUU. Recuperado de http://svaec. ifas.ufl.edu/docs/pdf/svreports/greenhousehydroponics/2003-04.pdf

Jasso-Chaverria, C., Hochmuth, G. J., Hochmuth, R. C., \& Sargent, S. A. (2005). Fruit yield, size, and color responses of two greenhouse cucumber types to nitrogen fertilization in perlite soilless culture. Hort Technology, 15(3), 565-571.

Johnny's Selected Seeds. (2014). Cucumber types and terminology. Recuperado de http://www.johnnyseeds.com/assets/information/cucumbers-types-terminology-8989. pdf

Kapuriya, V. K., Ameta, K. D., Teli, S. K., Chittora, A., Gathala, S., \& Yadav, S. (2017). Effect of spacing and training on growth and yield of polyhouse grown cucumber (Cucumis sativus L.). International Journal of Current Microbiology and Applied Sciences, 6(8), 299-304.

Lamb, E. M., Shaw, N. L., \& Cantliffe, D. J. (2001). Beit Alpha cucumber: a new greenhouse crop for Florida. Recuperado de http://www.hos.ufl.edu/protectedag/EDIS/CV27700. pdf

Musmade, A., \& Desai, U. (2003). Tratado de ciencia y tecnología de las hortalizas: el pepino y el melón. Zaragoza, España: Acribia.

Nomura, E. S., \& Cardoso, A. I. (2000). Redução da área foliar e o rendimento do pepino japonês. Scientia Agricola, 57(2), 257-261.

Patil, M. A., \& Bhagat, A. D. (2014). Yield response of cucumber (Cucumis sativus L.) to shading percentage of shade net. International Journal of Agricultural Engineering, 7(1), 243-248.

Premalatha, M. G., Wahundeniya, K. B., Weerakkody, W. A., \& Wicramathunga, C. K. (2006). Plant training and spatial arrangement for yield improvements in 
greenhouse cucumber (Cucumis sativus L.) varieties. Tropical Agricultural Research, 18, 346-357.

Rahil, M. H., \& Qanadillo, A. (2015). Effects of different irrigation regimes on yield and water use efficiency of cucumber crop. Agricultural Water Management, 148, 10-15.

Reche, J. (2011). Cultivo de pepino en invernadero. Madrid, España: Ministerio de Medio Ambiente y Medio Rural y Marino.

Sarhan, T. Z., \& Ismael, S. F. (2014). Effect of low temperature and seaweed extracts on flowering and yield of two cucumber cultivars (Cucumis sativus L.). International Journal of Agricultural and Food Research, 3(1), 41-54.

Shaw, N. L., Cantliffe, D. J., Funes, J., \& Shine III, C. (2004). Successful Beit Alpha cucumber production in the greenhouse using pine bark as an alternative soilless media. Hort Technology, 14(2), 289-294.

Shaw, N. L., Cantliffe, D. J., Rodríguez, J. C., Taylor, S., \& Spencer, D. M. (2000). Beit Alpha cucumber: an exciting new greenhouse crop. Proceedings of the Florida State Horticultural Society, 113, 247-253.

Soleimani, A., Ahmadikhah, A., \& Soleimani, S. (2009). Performance of different greenhouse cucumber cultivars (Cucumis sativus L.) in southern Iran. African Journal of Biotechnology, 8(17), 4077-4083.

Vasco, R. (2003). El cultivo de pepino bajo invernadero. In F. A. Camacho, Técnicas de producción en cultivos protegidos (Vol. Tomo 2, pp. 691-722). Madrid, España: Caja Rural Intermediterránea (Cajamar). 